\title{
Probing the Physics of Planets and Stars with Transit Data
}

\author{
Suzanne Aigrain \\ University of Oxford, Department of Physics, Keble Road, Oxford, OX1 3RH, UK \\ email: Suzanne.Aigrain@astro.ox.ac.uk \\ Invited Talk
}

\begin{abstract}
Summary. Virtually all exoplanet detection and characterisation methods are based on timedomain data. This invited talk gave an overview of some recent results in the field, highlighting some of the time-series-specific challenges encountered along the way. In particular it focussed on planetary transits: how to detect shallow, rare transits in noisy data, and how to model them with extreme accuracy to extract information about the transiting planet's atmosphere. Space-based transit surveys also constitute an extraordinary goldmine of information on stellar variability, and the talk touched briefly upon some recent statistical work in that field.
\end{abstract}

\begin{abstract}
Asteroseismology
Don Kurtz

Jeremiah Horrocks Institute, University of Central Lancashire, Preston, PR1 2HE, UK email: dwkurtz@uclan.ac.uk
\end{abstract}

Invited Talk

Summary. In 1926 in the opening paragraph of his now-classic book, The Internal Constitution of the Stars, Sir Arthur Eddington lamented, "What appliance can pierce through the outer layers of a star and test the conditions within?" While he considered theory to be the proper answer to that question, there is now an observational answer: asteroseismology. This talk introduced the concepts of asteroseismology, then looked at a selection of discoveries made for "ticking things" with the micromagnitude precision light curves of the KEPLER Mission. 